\title{
Audit on 'Appropriate periodontal management of new patients with a BPE of either 3 or $4^{\prime}$
}

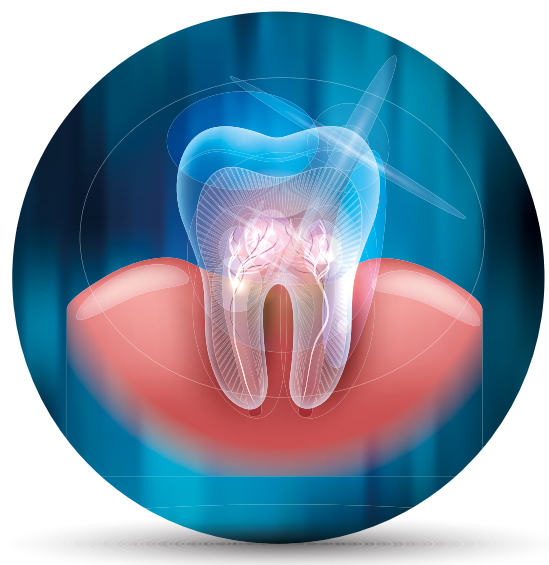

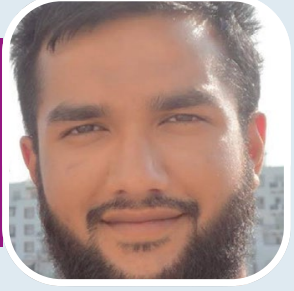

\section{By Ammar}

Ahmed

Zaki ${ }^{1}$

\section{Introduction}

Periodontal disease is one of the most common oral conditions in adulthood and is predominantly preventable. ${ }^{1}$ The prevalence of severe periodontal disease worldwide ranges around $10-15 \% .^{2}$ This is especially important to consider as misdiagnosis and mismanagement of periodontal disease can have a devastating outcome for patients ranging from pain, aesthetic concerns and even loss of dentition. ${ }^{3}$ It is also the leading cause for dental litigation in the UK, with $44.7 \%$ of all dental litigation in

\section{Author information}

'Ammar Ahmed Zaki completed a four-year MSci in integrated Physiology and Pharmacology for Research from King's College London in 2013. He went on to complete his BDS from the University of Liverpool in 2018, his PG Cert in teaching and learning in October 2020 and has passed the MFDS exams. He is currently carrying out his second year as a Dental Core Trainee in OMFS in North Wales. He is interested in pursuing a career in restorative dentistry and hopes to apply for speciality training in the coming years. He is also on $B D J$ Team's reader panel.
2015 being related to periodontal disease, and can therefore have an equally devastating effect for clinicians. ${ }^{4}$ It is of utmost importance that patients are aware of their condition, its causes and what can be done to manage the disease including available treatments.

The main risk factors for periodontal disease are poor oral hygiene, diabetes and tobacco use. ${ }^{5}$ As dental professionals, it is important to identify these risk factors as this allows for the provision of tailored advice to patients on how to manage their periodontal disease. As well as identifying risk factors, it is key that the patient is assigned the appropriate periodontal diagnosis. Often misdiagnosis occurs due to inadequate radiographs being present to assess bone levels and lack of evidence of a risk assessment. Mistreatment can also occur due to failure to monitor or treat periodontal disease appropriately. ${ }^{4}$

Considering the effects that periodontal disease can have on both the patient and the clinician, this audit was conducted to look at whether periodontal management of new patients who presented with a basic periodontal examination (BPE) score of 3 or 4 was appropriate.

\section{Standards}

The standard was set to a compliance level of $80 \%$ amongst the different practitioners within the practice. The British Society of Periodontology (BSP) is the main institution within the UK that provides guidance on the management of periodontal disease.

\section{Method}

A two-cycle audit was completed over a two one-month period, which were three months apart. Data were collected retrospectively through the completion of a case note review. New patients who had a BPE of 3 or 4 had their notes checked to see if all the relevant information has been recorded. The information gathered included:

- Presence of appropriate radiographs

- A recorded periodontal diagnosis

- A record of discussion of the diagnosis with the patient

- A record of discussion of the treatment plan with the patient

- If treatment was planned, carried out or rejected by the patient.

Four dental practitioners were included in this review. Notes for ten patients who had a BPE of 3 or 4 were looked at from each dentist, making the total sample size 40 per cycle. This sample size was chosen as it allowed for a manageable data collection process while still allowing enough data points for analysis.

First audit cycle results and discussion

For the $1^{\text {st }}$ cycle data were collected retrospectively from the month of February 2019 using the computerised notes on Software of Excellence (SOE). The $1^{\text {st }}$ cycle was randomised without any of the stakeholders being informed that the audit was being carried out. The questions that were asked and the overall average for the team can be seen in Table 1.

In the $1^{\text {st }}$ cycle, the set standard was not met on all parameters assessed. Practitioners overall performed better in the areas of appropriate radiographs and recording 
and discussing the diagnosis. However, practitioners fell significantly below standard in areas that included discussing and implementing a treatment plan with a patient. The overall pooled average was $61 \%$ falling $19 \%$ below the set standard.

\section{Implementation of changes}

In order to eliminate or reduce the patient's risk of misdiagnosis and mistreatment of periodontal disease as well as reduce the practice's risk to litigation for periodontal related issues, it was decided that it was important to implement a practice wide strategy that would help reach the $80 \%$ compliance level set in this audit and improve periodontal disease management. The following changes were implemented:

1. During a practice wide meeting the results of the first cycle were discussed, giving an opportunity to provide a refresher on the current guidance and for any questions.

2. Next to the computer screens in each surgery the below were placed (this area was chosen so practitioners can see them clearly when they write up the patient's notes):

a. New Periodontal classification: to allow for accurate recording of diagnosis

b. Flow chart from the BSP with the required steps for each diagnosis: So correct treatment was prescribed for each patient

3. Free text note templates were made on SOE for the following:

a. Exam appointment

b. Treatment appointment

4. New periodontal diagnosis software addition was installed onto SOE, to help practitioners record the correct periodontal diagnosis.

Recollection of data occurred in May 2019.

\section{Second audit cycle results and discussion}

For the $2^{\text {nd }}$ cycle data were collected retrospectively from the month of May 2019 using the same method as the first cycle to allow comparison of the results. The questions that were asked and the overall average for the team can be seen in Table 2 .

In the $2^{\text {nd }}$ audit cycle, it can clearly be seen that the rate of compliance overall improved for all dentists. Overall, the practice reached the set standard of $80 \%$. The compliance improved from $61 \%$ to $83 \%$. There are still areas that did not hit the $80 \%$ compliance which included the recording and discussion of a diagnosis.

Table 1 Questions asked in the 1st cycle and averages

\begin{tabular}{l|l} 
Questions & \multicolumn{2}{c}{ Overall average \% } \\
\hline Were the appropriate radiographs present or taken? & 70 \\
\hline Was the diagnosis recorded? & 65 \\
\hline Was the diagnosis discussed with the patient and recorded? & 65 \\
\hline Was the treatment discussed with patient and recorded? & 48 \\
\hline Was the treatment planned, carried out or rejected by the patient? & 55 \\
\hline Overall average & $\mathbf{6 1}$ \\
\hline
\end{tabular}

Table 2 Questions asked in the 2nd cycle and averages

\begin{tabular}{l|l} 
Questions & \multicolumn{2}{l}{ Overall average \% } \\
\hline Were the appropriate radiographs present or taken? & 95 \\
\hline Was the diagnosis recorded? & 73 \\
\hline Was the diagnosis discussed with the patient and recorded? & 70 \\
\hline Was the treatment discussed with patient and recorded? & 85 \\
\hline Was the treatment planned, carried out or rejected by the patient? & 90 \\
\hline Overall average & $\mathbf{8 3}$ \\
\hline
\end{tabular}

\section{Conclusion}

In conclusion, this audit has successfully managed to improve the rate of compliance amongst the clinicians within the practice. Clinicians are aware of guidelines provided and can tailor their treatment plans as such, which ultimately helps in making better decisions for the appropriate and safe management of periodontal disease. This in turn improves patients' standard of care, and helps to prevent the loss of teeth.

Ideally $100 \%$ of patients should have the appropriate treatment plan prescribed for them as this would mean that all patients are receiving the correct level of dental care. The other dental practitioners within the practice felt that the main reason for not being able to achieve this is fatigue and time pressure. A limitation of this audit is the sample size as only data from ten patients per dentist were recorded which was 40 patients in total. There is a possibility that such a sample size could give a skewed result.

Periodontal disease diagnosis and treatment is ultimately a shared responsibility between dentist, dental care professionals and the patients. Many patients are unaware of periodontal disease and its implications on their oral condition. Simple steps can be implemented to avoid mismanagement of periodontal disease including explaining the diagnosis to the patient, constructing a long-term plan with the patient and documenting it. Documenting the discussions is particularly important as the management of periodontal disease can be lifelong for a lot of patients. Having previous management documented allows clinicians to refer back and see what was effective in the past. It is also important that, if working under a prescription by a dentist, this is adequate and satisfies the BSP guidelines. If this is not the case, then it should be discussed with the prescribing dentist prior to commencement of management and treatment. The new classification allows the ability to reach a diagnosis and lets the clinician know exactly where the patient is within the disease cycle, and this can help grant patients the best possible treatment to prevent future tooth loss.

\section{References}

1. Nazir M A. Prevalence of periodontal disease, its association with systemic diseases and prevention. Int $J$ Health Sci 2017; 11: 72-80.

2. Meyer M S, Joshipura K, Giovannucci E, Michaud D S. A review of the relationship between tooth loss, periodontal disease, and cancer. Cancer Causes Control 2008; 19: 895-907.

3. Ridgeway E E. Periodontal disease: diagnosis and management. J Am Acad Nurse Pract 2000; 12: 79-84.

4. Wadia R. Reena's Notes: How to Avoid Periodontal Litigation in General Dental Practice with Dr Ian Dunn. 2018.

Available at: https://www.reenawadia.com/ reenas-notes-how-to-avoid-periodontallitigation-in-general-dental-practice-withdr-ian-dunn/ (accessed March 2021).

5. Genco R J, Borgnakke W S. Risk factors for periodontal disease. Periodontology 2000 2013; 62: 59-94.

https://doi.org/10.1038/s41407-021-0585-0 